\title{
FEM BASED MODELING OF CYLINDRICAL-MAGNETIC ABRASIVE FINISHING (C-MAF) PROCESS USING UNBOUNDED MAGNETIC ABRASIVES
}

\author{
K. B. Judal \\ Mechanical Engineering Department, \\ Government Engineering College Patan, Patan-384265, Gujarat, India \\ Email: judalkb@yahoo.com
}

\begin{abstract}
Cylindrical magnetic abrasive finishing (C-MAF) process is an advanced abrasion based finishing technique used for precision finishing of external and internal surfaces of rotational jobs. Flexible brush of unbonded magnetic abrasives formed in the working gap which gently finishes the surface irregularities. This paper focuses on theoretical investigations of the C-MAF process. A finite element model is developed to evaluate the distribution of magnetic forces on the workpiece surface. The process removes a very small amount of material by indentation of abrasive particles and rotation of the workpiece. A theoretical model for material removal and surface roughness is also proposed considering a uniform triangular surface roughness profile. The simulation results are verified by comparing them with the experimental results available in the literature. The saturation in surface roughness is also simulated with the help of critical surface roughness value at particular magnetic flux density.
\end{abstract}

Keywords-modeling; MAF; unbonded magnetic abrasives, FEM; abrasion; surface roughness; finishing

\section{INTRODUCTION}

Magnetic abrasive finishing (MAF) is an advanced abrasion based precision finishing technique in which magnetic abrasive particles (MAPs) are magnetized due to magnetic flux present in the working gap and form a flexible brush like multi-point cutting tool that produces abrasion due to relative motion with the surface of the workpiece and gently finishes it. MAPs are prepared by mixing ferromagnetic particles (e.g. Iron particles, steel grit, etc.) with abrasives ( $\mathrm{SiC}, \mathrm{Al}_{2} \mathrm{O}_{3}, \mathrm{Cr}_{2} \mathrm{O}_{3}$, diamond powder etc.). MAPs can be used as bonded, unbonded or loosely bonded [1]. Bonded magnetic abrasives are prepared by sintering ferromagnetic particles and abrasives at very high pressure and temperature in an inert gas atmosphere and then crushed and sieve to specific size. Each bonded MAP contains ferromagnetic part with abrasives embedded in it (Fig.1a). Unbonded magnetic abrasives (UMAs) are prepared by simple mechanical mixing of ferromagnetic particles and abrasive particles (Fig.1b) [2]. Due to non-magnetic behavior, abrasives flown away during dry machining or washed away if coolant is supplied. In order to reduce the problems associated with UMAs the gel or oil is mixed with UMAs which is called loosely bonded magnetic abrasives [3].

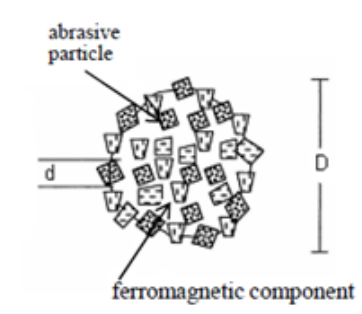

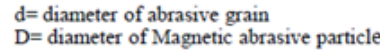
(a)

Fig. 1. abrasives

The abrasion pressure required for finishing can be controlled by varying the magnetic field in the working gap. Magnetic field can be generated either by electromagnet or permanent magnet. Electromagnet is best suited for the process because abrasion pressure developed can be easily controlled by varying current to the electromagnet. The disturbances from the machine structure due to vibration or chatter will not affect the quality of the finished surface. This process has following advantages over conventional finishing processes [4]

- Material surface is free from burns and thermal defects

- Non-ferrous materials can also be finished

- Simple in implementation

- Any complex and contoured surfaces can be finished

MAF can be applied to finish external and internal surfaces of rotational jobs as well as plane surfaces. Based on its application, MAF can be categorized into: cylindrical-MAF (C-MAF) and Plane MAF. C-MAF can be used to produce nano-level surface finish on shafts and rods used in sliding pairs to reduce power loss in friction and increase the life of the components.

During C-MAF, the relative motion between the flexible brush and workpiece surface due to rotation of the workpiece is responsible for material removal particularly from the peaks of the surface irregularities. In case of UMAs abrasives are randomly distributed. The primary cutting/scratching is performed by abrasives while ferromagnetic particles (FPs) supplies abrasion pressure. The number of abrasives takes part in cutting action beneath each FP may vary from one instance to another instance. Hence, the C-MAF process with UMAs is not completely understood as the quantitative relationship between process parameters (viz. magnetic flux density (MFD), size of FPs, size of abrasive grain, workpiece 
rotational speed, etc) and process performance is not predicted.

From the literature review, it is also found that mainly experimental investigations have been carried out by various researchers on C-MAF. Hence, there is a need to expand scientific and technical knowledge of the process to improve the overall performance of the process and achieve wider acceptance in the manufacturing industries. Modeling of the C-MAF process would help in understanding the principle of the process and mechanism of material removal. This would also results in elucidating the effect of various parameters on the material removal and surface finish without conducting actual experiments.

\section{MECHANISM OF MATERIAL REMOVAL}

In order to understand the mechanism of material removal during C-MAF, it is necessary to concentrate on distribution of magnetic field between the magnetic pole and workpiece. Fig. 2(a) shows the distribution of magnetic field in twodimensional system and forces acting on FP at different locations within the working gap [5]. The FPs in the UMAs combined to each other magnetically between magnetic pole and workpiece along the lines of magnetic force forming a flexible brush like tool. When FP is considered at position A, near the work surface, the normal force $F_{N}$ that pushes the FP and abrasives on the work surface to indent it. Due to rotary motion of the workpiece the cutting resistance, $R_{T}$ is acting in tangential direction tends to move the FP from its balanced position. The tangential force $F_{T}$ due to magnetic field, acts in opposite direction of cutting force $R_{T}$, returns the FP to its original position [6]. It shows that FP will transmit abrasion pressure to abrasives beneath it and abrasives will perform a regular cutting on the work surface. Due to regular cutting, the material will be removed in the form of micro-chips as shown in Fig. 2(b). For very hard workpiece, $R_{T}$ will be more than the $F_{T}$ and FP begins to roll. It decreases the cutting efficiency of the abrasive grain. When $F_{T}$ is very small FP splash out from the working gap and no cutting will takes place. Due to the gradient of magnetic field the ferromagnetic particles in the brush near the workpiece surface presses the abrasive grains on the surface and cutting edge of abrasive is capable of infinitesimal cutting. The $F_{N}$ and $F_{T}$ can be derived from the forces acting on the FP along the lines of magnetic force $\left(F_{x}\right)$ and along magnetic equipotential lines $\left(F_{y}\right)$. When FP is at position $\mathrm{B}$ outside the working gap, the forces $F_{x}$ and $F_{y}$ tends to concentrate the FP within the working gap and prevents the splashing of the FP when workpiece is rotated.

\section{MODELING OF MATERIAL REMOVAL}

A magnetic potential distribution in the two dimensional domain was calculated using finite element method (FEM) to find the forces acting on the FPs at the workpiece-brush interface to find the material removal during MAF. The surface roughness model was developed by considering total (a)
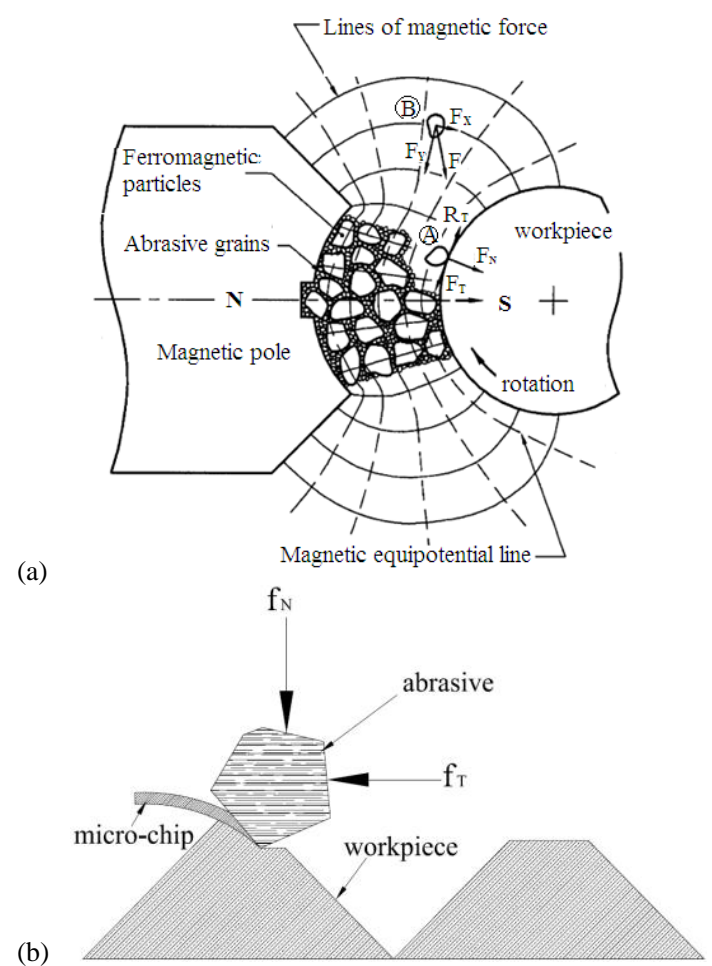

Fig. 2. (a) Two-dimensional magnetic field distribution (b) cutting action of single abrasive grain

volume of material removed with the assumption of triangular surface profile without statistical considerations. Following sections describe the step-by-step procedure for development of UMA based C-MAF process model.

\section{A. Finite Element Formulation of Magnetic Potential}

The physical phenomena of the C-MAF process can be described by boundary value problem. Due to the complex and unpredictable nature of C-MAF process while using UMAs, as well as it involves intricate geometry of solution domain as represented in Fig. 3(a) and composite material properties, the use of analytical methods to obtain the solution of the governing equation under such conditions would be very difficult. FEM can be used to obtain the approximate numerical solution of such complicated C-MAF problem [7].

\section{Mathematical Modeling}

The FPs among the UMAs acquire magnetic polarization and join each other along the lines of magnetic force and forming a flexible brush like multi-point cutting tool. To determine the distribution of magnetic forces the governing equation of the process is expressed in terms of the magnetic potential, which is primary variable. Following assumptions are made to simplify the C-MAF process model:

1. The intensity of magnetic field is not varying with time.

2. Leakage of magnetic flux from the domain is neglected.

3. The UMAs are closely packed in the form of brush between each pole and workpiece.

4. The micro chips removed during MAF process have insignificant effect on the properties of flexible brush. 
5. The relative permeability of UMAs is calculated by considering relative volume fractions of ferromagnetic particles and abrasive particles.

6. Solution domain is two dimensional, i.e. $\frac{\partial \varphi}{\partial Z}=0$.

The steady state Laplacian form of governing equation within two dimensional domain (Fig. 3a) can be written as [8],

$\frac{\partial}{\partial X}\left(\mu_{r} \frac{\partial \varphi}{\partial X}\right)+\frac{\partial}{\partial Y}\left(\mu_{r} \frac{\partial \varphi}{\partial Y}\right)=0$ in the domain

where, $\mu_{r}$ is the relative permeability of UMAs.

Boundary Conditions:

There are total four numbers of boundaries: $B_{1}, B_{2}, B_{3}$ and $B_{4}$.

(1) Essential boundary conditions

The scalar magnetic potential $\varphi$ is specified on the boundaries $B_{1}$ and $B_{2}$. Here there is no current source in the domain, so the problem is solved based on reduced scalar potential strategy. If the magnetic field is generated by means of electromagnet having $N_{m}$ number of turns through which $I_{m}$ current is flowing, then

$$
\varphi=k_{m} N_{m} I_{m} \quad \text { on } B_{l}
$$

where, $k_{m}$ represents the efficiency of electromagnet to consider the loss of magnetic flux at different current to electromagnet. The value of $k_{m}$ can be determined by numerical computation of MFD generated at particular point in the working gap and experimental measurement of MFD at same location by gauss meter at different current to electromagnet.

$$
\varphi=0 \quad \text { on } B_{2}
$$

(2) Natural boundary conditions

In case of non-magnetic workpiece, all magnetic field lines may not pass through the workpiece, but some field lines may flare and passing from $\mathrm{N}$-pole to S-pole through air. While for magnetic workpiece, the field lines follow the less reluctant path. On boundary $B_{3}$ and $B_{4}$, the normal derivative of the scalar potential is zero.

$$
\frac{\partial \varphi}{\partial n}=0 \quad \text { n } B_{3} \text { and } B_{4}
$$

Finite element method [9] is used to evaluate magnetic potential distribution within the solution domain. A part of the discretized solution domain is shown in Fig.3(b). Galerkin's finite element approach is applied for solution of governing equation (Eq.1) and boundary conditions (Eq.2-4). For each typical element (Fig. 3c) in the solution domain, an elemental equation has been developed. The elemental equation over a typical element is given by,

$$
[K]^{e}\{\varphi\}^{e}=0
$$

where, $[K]^{e}$ is the elemental coefficient matrix and $\{\varphi\}^{e}$ is magnetic scalar potential vector of the respective element. The elemental coefficient matrix is given by,

$$
[K]^{e}=\int_{A_{e}} \mu_{r}[B]^{e T}[B]^{e} d X d Y
$$

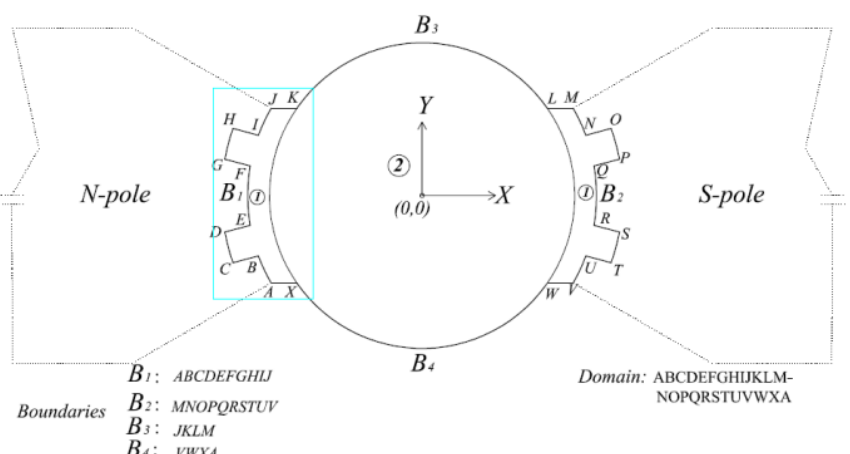

(a)

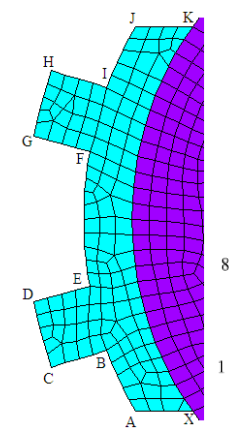

(b)

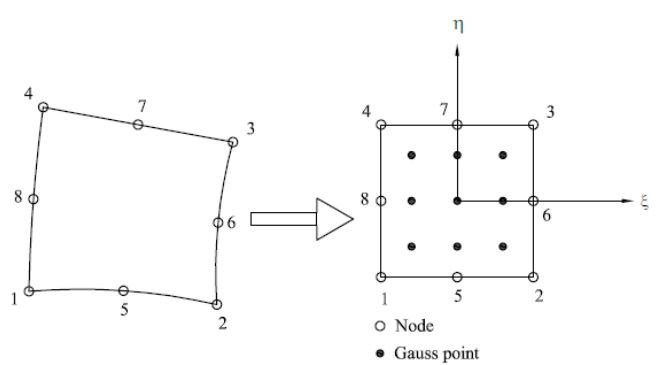

(c) (d)
Fig. 3. (a) Solution domain of MAM (b) portion of discretized domain (c) typical eight-noded quadrilateral element (d) square master element

The $[B]^{e}$ represents the matrix of derivatives of shape functions for a corresponding element. Then, the global coefficient matrix $[G K]$ is obtained after assembling the elemental coefficient matrices over all the elements. The solution leads to global equation as given in Eq.7,

$[G K]\{\varphi\}=\{0\}$

The Eq.7 is a set of linear algebraic equations. These equations are solved by simultaneous equation solver after imposing essential boundary conditions. Solution leads to nodal values of magnetic scalar potential $(\varphi)$ at all nodes in the domain.

\section{B. Calculation of Cutting Forces}

From the nodal values of $\phi$, the values of magnetic field intensity $(\mathrm{H})$, and gradients of magnetic field intensity $\left(\frac{d H}{d X}\right.$ and $\left.\frac{d H}{d Y}\right)$ are calculated at Gauss points near the workpiece contact surface in region (1) (Fig.3a) to compute the forces acting on ferromagnetic particle (FP) along $\mathrm{X}$ and $\mathrm{Y}$ directions. The forces acting on a FP in the magnetic field is given by [10],

$$
F_{X}=\chi_{F P} \mu_{0} V H\left(\frac{d H}{d X}\right) \& F_{Y}=\chi_{F P} \mu_{0} V H\left(\frac{d H}{d Y}\right)
$$

where, magnetic field intensity $H=-\nabla \varphi, \mu_{0}=$ permeability of free space, $\mathrm{V}=$ volume of FP, $\chi_{F P}=$ susceptibility of FPs Here, the UMAs consist of simple mechanical mixture of FPs and abrasives. The relative permeability of UMAs $\left(\mu_{r}\right)$ can be calculated as [11]; 
$\mu_{r}=\alpha \mu_{F P}+(1-\alpha) \mu_{A B R} \quad$ and $\quad \chi_{F P}=\mu_{F P}-1$

where, $\alpha=$ volume fraction of FPs in UMAs

$$
\begin{aligned}
& \mu_{A B R}=\text { relative permeability of abrasives } \\
& \mu_{F P}=\text { relative permeability of FPs }
\end{aligned}
$$

The contact surface between the workpiece and flexible magnetic abrasive brush is represented by arc of circles which is the common portion of regions (1) and (2) (Fig.3a). The values of $\mathrm{H}$ and its derivatives are more accurate at Gauss points than at nodes. Therefore, the approximate length of the mess element is selected as a function of size of FP, so that the center of each FP at contact surface approximately lies on the Gauss point.

The cutting forces $F_{N}$ and $F_{T}$ acting on steel grit can be calculated by resolving the forces, $F_{X}$ and $F_{Y}$ along normal N$\mathrm{N}$ and tangent T-T directions as shown in Fig.4(a) [12].

$F_{N}=F_{X} \cos \theta+F_{Y} \sin \theta \& \quad F_{T}=-F_{X} \sin \theta+F_{Y} \cos \theta$

Both $F_{N}$ and $F_{T}$ are spatial in nature as there magnitude and direction varies with location of FP in two-dimensional system.

\section{Calculation of Material Removal}

Abrasives are randomly distributed in the UMAs. It is assumed that primary cutting action is performed by abrasive particles and FPs provide abrasion pressure due to normal force. The number of abrasive particles take part in abrasion action beneath each FP may vary from one instance to another instance. Based on probability statistics function the actual number of contacting grains may vary from $3.8 \%$ to $18 \%$ of the total number of abrasive particles per unit area [14]. Considering these aspects, the number of abrasive particles under each FP is selected by random permutation of numbers.

Let, $\mathrm{n}_{\mathrm{a}}$ is the number of abrasive particles under FP at an instance as captured from random permutation.

Normal force acting on single abrasive particle responsible for indentation is given by,

$$
f_{N}=\frac{F_{N}}{n_{a}}
$$

The abrasive particles have angular cutting edges which can be considered as wedge shape with included angle $2 \theta_{a}[15]$. The indentation depth of such wedge-shaped abrasive at a particular instance can be determined from Fig. 4(b).

$$
f_{N}=H_{m t} \pi a^{2}
$$

where, $a=t_{p} \tan \theta_{a}, H_{m t}=$ hardness of workpiece surface, $a=$ radius of projected area of indentation, $t_{p}=$ depth of penetration of abrasive particle in workpiece surface.
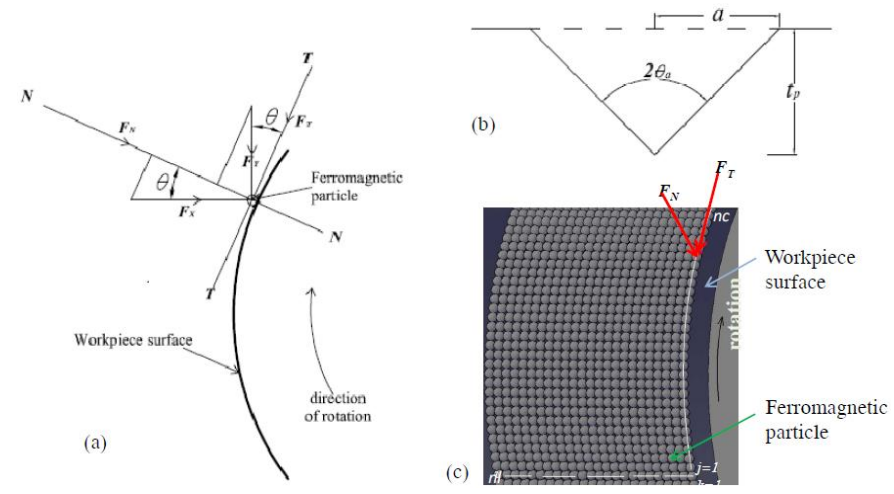

Fig. 4. Forces acting on ferromagnetic particle during MAF (b) Indentation by wedge shaped abrasive particle (c) Grid of ferromagnetic particles at contact surface. [13]

The cross-sectional area of penetration by abrasive particle is given by,

$$
A_{a}=t_{p}^{2} \tan \theta_{a}
$$

Fig. 4(c) shows the grid of FPs at contact surface of the cylindrical workpiece within the flexible magnetic abrasive brush during MAF. Each FP provides normal force on the abrasive particle beneath it. The normal force, $f_{N}(j)$ acting on abrasive particle depends on the location of FP in twodimensional system (X-Y plane) and number of abrasive cutting edges $\left(n_{a}\right)$ simultaneously penetrating the workpiece surface. Let, $n l$ and $n c$ are number of FPs simultaneously in contact with surface along workpiece length and circumferential direction respectively as shown in Fig.4(c). By considering the workpiece surface as uniform triangular profile without statistical distribution, the total amount of material removed by MAF $\left(V_{M A F}\right)$ from the surface within given machining time can be calculated as follow:

$$
V_{M A F}=K_{\text {maf }} v_{r e l} T_{m}\left(1-\frac{R_{a}}{R_{a}^{0}}\right) \sum_{k=1}^{n l} \sum_{j=1}^{n c}\left(\frac{n_{a} f_{N}(j)}{H_{m t} \pi \tan \theta_{a}}\right)
$$

where, $K_{\text {maf }}$ is the constant for MAF which depends on number of factors as MAF can be considered as the case of three-body abrasion system, $v_{\text {rel }}$ represents the relative velocity of abrasive particles at the workpiece surface, $R_{a}^{0}$ and $R_{a}$ represent initial and final arithmetic mean surface roughness respectively.

$$
K_{\text {maf }}=\frac{k^{\prime} k_{0} k_{1} k_{2} k_{3} k_{4}}{k_{5}}
$$

Wang and Wang [15] have recommended various criteria to consider different factors and their values in case of threebody abrasion. In the case of UMAs the value of different factors in Eq.15 are given in Table I along with consideration. 


\section{MODELING OF SURFACE ROUGHNESS}

Following assumptions are taken for easy modeling of surface roughness during $\mathrm{C}-\mathrm{MAF}$ process.

TABLE I. VALUE OF DIFFERENT FACTORS WHILE CALCULATING THE $K_{M A F}[15]$.

\begin{tabular}{llll}
\hline Factor & \multicolumn{1}{c}{ Consideration } & Value \\
\hline$k^{\prime}$ & $\begin{array}{l}\text { proportionality constant which is more or less } \\
\text { constant }\end{array}$ & 0.03 \\
$k_{0}$ & $\begin{array}{l}\text { Hardness of workpiece and steel grit is } \\
\text { approximately same }\end{array}$ & 0.50 \\
$k_{1}$ & $\begin{array}{l}\text { Abrasives are closely packed between steel grit and } \\
\text { workpiece surface }\end{array}$ & 1.0 \\
$k_{2}$ & $\begin{array}{l}\text { Material of the workpiece is steel } \\
k_{3}\end{array}$ & $\begin{array}{l}\text { Electrolyte containing water is always present in the } \\
\text { form of lubrication between abrasive and workpiece } \\
\text { surface }\end{array}$ & 0.67 \\
$k_{4}$ & $\begin{array}{l}\text { Ductility of the workpiece material } \\
\text { Relative hardness of workpiece material and } \\
k_{5}\end{array}$ & $\begin{array}{l}\text { abrasives, here hardness of SiC abrasive is greater } \\
\text { than two times hardness of workpiece }\end{array}$ & 1.0 \\
\end{tabular}

1. Initial surface roughness having uniform triangular profile (Fig. 5).

2. The valleys are inaccessible to magnetic abrasive brush and major amount of material removal takes place from the peaks of the surface profile.

3. Abrasive grains in UMAs move in a direction perpendicular to the length of triangular profile (Fig. 5).

4. After all peaks of irregularities have been removed, further material removal does not contribute to improvement in surface finish.

Let the workpiece surface has initial arithmetic mean surface roughness, $R_{a}^{0}$. After, processing for time $T_{m}$ during

C-MAF process, the final surface roughness achieved is $R_{a}$. For uniform triangular profile, the maximum peak to valley height $=4 R_{a}^{0}$ [16]. In this work the final surface roughness obtained can be calculated from the total volume of material removed during C-MAF process (Eq. 14). The major amount of material is removed from the peaks of the surface profile and the peaks get truncated (Fig. 6). From the geometry, the total amount of material removed from the periphery of the cylindrical workpiece on machining length $\left(l_{w}\right)$ can be written as follow.

$$
V_{w}=\frac{\pi d_{w}}{2}\left(1-\frac{R_{a}}{R_{a}^{0}}\right) \times 4\left(R_{a}^{0}-R_{a}\right) \times l_{w}
$$

Here, the surface profile is assumed uniform triangular but in real case the profile is random in nature and the ratio of maximum peak-valley height to $R_{a}^{0}$ may vary from 5 to 10 for grounded surfaces [16]. Therefore, above equation can be modified by incorporating constant $\left(k_{r}\right)$, which takes care of initial surface roughness profile of the workpiece surface.

Final arithmetic means surface roughness; $R_{a}$ can be given by following equation,

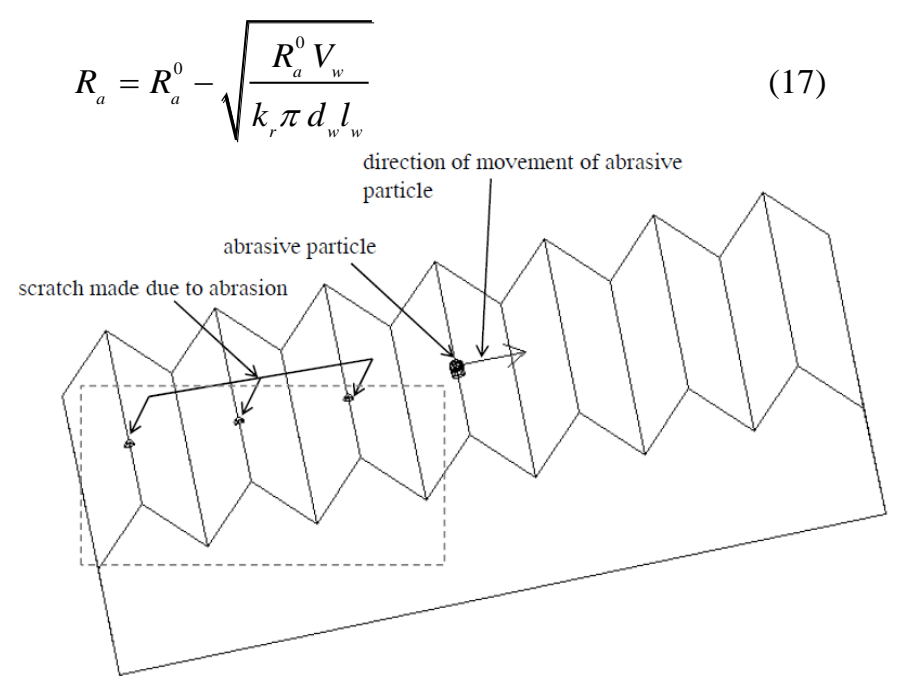

Fig. 5. Schematic diagram of developed workpiece surface profile and cutting action of abrasive

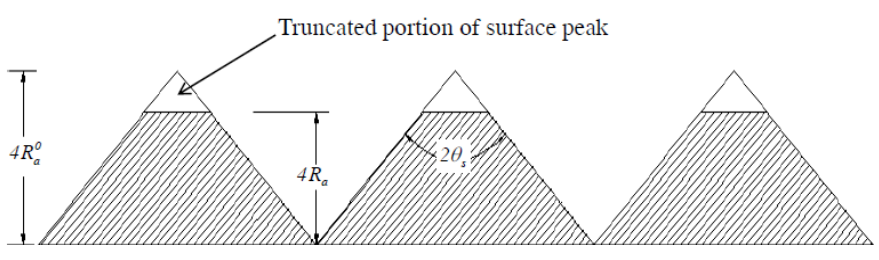

Fig. 6. Cross-section of cut-portion of surface profile peaks

From the above equation the final surface roughness of the workpiece can be calculated if the total volume of material removed from the machining zone is known. But, the critical value of surface roughness at each value of current to electromagnet beyond which further roughness reduction is not possible can be determined by

$$
R_{a}=\frac{t_{p(\text { mean })}}{2}
$$

The higher value of $R_{a}$ obtained from both the Eq. (17) and Eq. (18) is taken as a final surface roughness.

\section{VALIDATION OF C-MAF PROCESS MODEL}

In the present work, a MATLAB code has been developed for modeling of material removal and surface roughness during C-MAF process. Based on the convergence study of the mesh for magnetic potential distribution and size of FPs, the domain is discretized into suitable number of eight noded quadrilateral isoparametric elements (Fig. $3 \mathrm{c} \& d$ ) for calculation of normal and tangential cutting force at contact surfaces. The inputs applied to the program are initial surface roughness, current to electromagnet and workpiece rotational speed and the outputs obtained from the program are MFD in the working gap at specified locations, material removal and surface roughness obtained after specified machining time, $T_{m}$.

The simulation results for material removal and surface roughness during $\mathrm{C}-\mathrm{MAF}$ are validated with the experimental results of Yan et al. [17]. The machining parameters and material properties employed in the simulation are taken from reference [17] and are given in Table II. The simulated material removal and surface roughness with respect to 
machining time are plotted on common graph. The experimental results are also superimposed on the same graph

TABLE II. PROCESS CONDITIONS FOR VALIDATION OF C-MAF MODEL [17]

\begin{tabular}{|c|c|c|}
\hline Working gap & \multicolumn{2}{|l|}{$: 1.0 \mathrm{~mm}$} \\
\hline Magnetic flux density & \multicolumn{2}{|l|}{$: 0.85 \mathrm{~T}$} \\
\hline Workpiece rotational speed & \multicolumn{2}{|c|}{ : 500 RPM, 800 RPM } \\
\hline Type of magnetic abrasives & \multicolumn{2}{|c|}{ :Unbonded magnetic abrasive particles (UMAs) } \\
\hline Ferromagnetic particles (FPs) & \multicolumn{2}{|c|}{ : Steel grit (grit size: $180 \mu \mathrm{m})$} \\
\hline Abrasives & \multicolumn{2}{|c|}{ : Silicon Carbide (grit size $1.2 \mu \mathrm{m}$ ) } \\
\hline \multicolumn{3}{|c|}{ Number of FPs involved in machining $\quad: n l=320, n c=55$} \\
\hline \multicolumn{3}{|c|}{$\begin{array}{l}\text { Number of abrasive grains under each FP } \\
\text { permutation }\end{array}$} \\
\hline \multicolumn{3}{|c|}{ Average semi-angle of conical abrasive $\quad: 70^{\circ}$} \\
\hline Workpiece material & \multicolumn{2}{|c|}{ : SKD 11 tool steel } \\
\hline Diameter of workpiece & \multicolumn{2}{|c|}{ : $15 \mathrm{~mm}$} \\
\hline Finishing length & \multicolumn{2}{|l|}{ : $65 \mathrm{~mm}$} \\
\hline Hardness of the workpiece & \multicolumn{2}{|l|}{ : $7050 \mathrm{MP}_{\mathrm{a}}$} \\
\hline Initial arithmetic mean surfac & roughness & $0.18 \mu \mathrm{m}$ \\
\hline
\end{tabular}
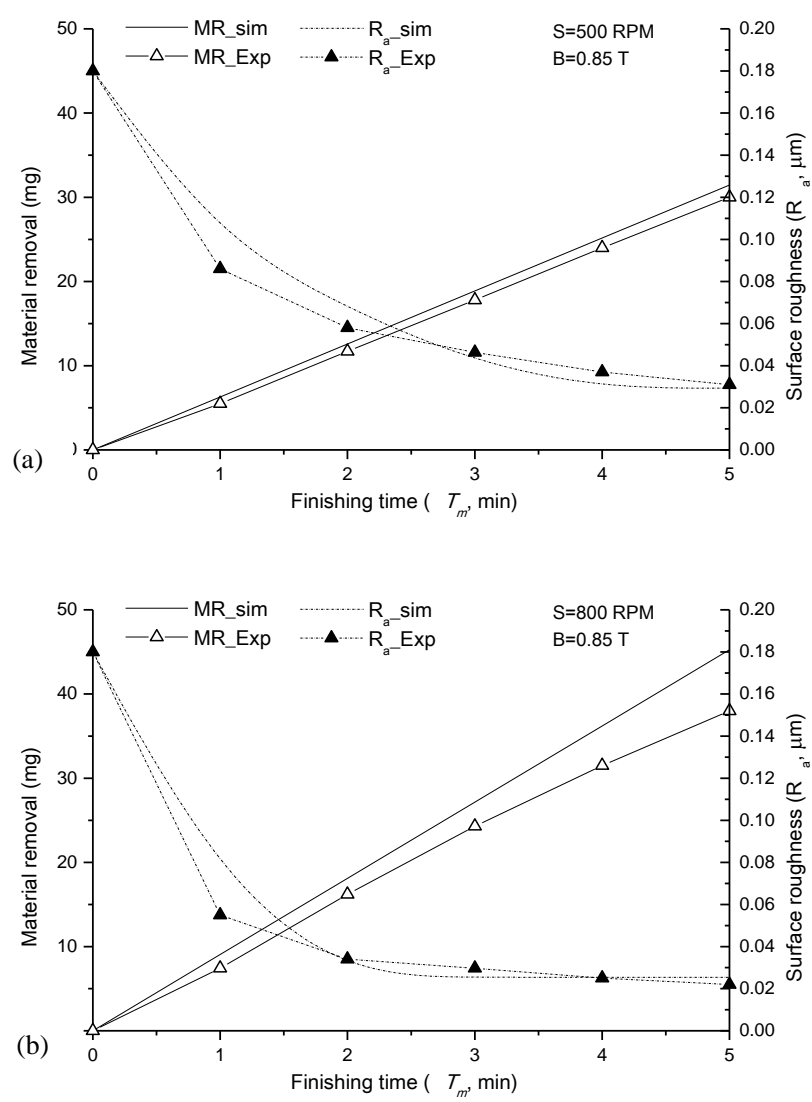

Fig. 7. Validation of simulation results of C-MAF performance with experimental results (a) $500 \mathrm{RPM}$ (b) $800 \mathrm{RPM}[17]$

for comparison. Fig. 7(a) and Fig. 7(b) show the performance of C-MAF for workpiece rotational speed 500 RPM and 800 RPM respectively. It is observed that the simulated material removal and surface roughness follow the same trend as the experimental results and are quite close to actual results. The simulated results of surface roughness also shows saturation tendency like experimental results after $2.5 \mathrm{~min}$. of finishing during C-MAF at $800 \mathrm{RPM}$ as shown in Fig. 7(b). The saturation in surface roughness is computed considering the critical value of surface roughness at given MFD. This validates the modeling of C-MAF.

\section{CONCLUSIONS}

A FEM based C-MAF process model for prediction of material removal and surface roughness has been proposed. This helps in understanding the mechanism of forces applied and the material removal. The simulation results of material removal and surface roughness with respect to time are validated with previously published experimental data and are in good agreement. The saturation of surface roughness observed in experimental results is also simulated with the help of critical surface roughness at particular MFD.

\section{References}

[1] V.K. Jain, P. Kumar, P.K. Behera, and S.C. Jayswal, "Effect of working gap and circumferential speed on the performance of magnetic abrasive finishing process," Wear, vol. 250, pp. 384-390, 2001.

[2] K.B. Judal, and V. Yadava, "Review of research work in magnetic abrasive finishing," Proceedings of National Conference on Recent Advances in Manufacturing (RAM-2010),SVNIT Surat, pp. 59-65, 2010

[3] A.C. Wang, L. Tsai, C.H. Liu, K.Z. Liang, and S.J. Lee, "Elucidating the optimal parameters in magnetic finishing with gel abrasive," Mater. Manuf. Process.vol 26, pp. 786-791, 2011

[4] P. Jayakumar, S. Ray, and V. Radhakrishnan, "Optimising process parameters of magnetic abrasive machining to reduce the surface roughness value,”. J. Spacecraft Technol.vol. 7, pp. 58-64, 1997.

[5] T. Shinmura, K. Takazawa, and E. Hatano, "Study on magnetic abrasive finishing- effect of various types of magnetic abrasives on finishing characteristics,” Bull. Jpn. Soc. Precis. Eng. 21, 2 (1987), 139-141.

[6] T. Mori, K. Hirota, and Y. Kawashima, "Clarification of magnetic abrasive finishing mechanism," J. Mater. Process. Technol. Vol. 143144, pp. 682-686, 2003.

[7] G. Dhatt and G. Touzot, The Finite Element Method Displayed. John Wiley and Sons, Chichester, UK, 1984.

[8] T.R. Chandrupatla and A.D. Belegundu, Introduction to Finite Elements in Engineering. PHI Learning Private Limited, New Delhi, 2008.

[9] J.N. Reddy, An Introduction to the Finite Element Method. Tata McGraw-Hill Publishing Company Limited, New Delhi, 2005.

[10] M.R. Smolkin, and R.D. Smolkin, "Calculation and analysis of the magnetic force acting on a particle in the magnetic field of separator. Analysis of the equation used in the magnetic methods of separation," IEEE Trans. Magn. vol. 42, pp. 3682-3693, 2006.

[11] S.C. Jayswal, V.K. Jain, and P.M. Dixit, "Modeling and simulation of magnetic abrasive finishing process," Int. J. Adv. Manuf. Technol. vol. 26, pp. 477-490, 2005.

[12] K.B. Judal, and V. Yadava, "Modeling and simulation of cylindrical electro-chemical magnetic abrasive machining process," Machin. Sci. Technol. vol.18, pp. 221-250, 2014.

[13] K.B. Judal, and V. Yadava, "Modeling and simulation of cylindrical electro-chemical magnetic abrasive machining of aisi-420 magnetic steel,” J. Mater. Process. Technol. vol. 213, pp. 2089-2100, 2013.

[14] Z.B. Hou,and R.Komanduri,"Magnetic field assisted finishing of ceramics-part III: on the thermal aspect of magnetic abrasive finishing (MAF) of ceramic rollers," ASME J. Tribol. vol. 120, pp.660-667, 1998.

[15] Y.L. Wang, and Z.S. Wang, "An analysis of the influence of plastic indentation on three-body abrasive wear of metals," Wear vol. 122, pp. $123-133,1988$.

[16] Tokyo Seimitsu Co Ltd, Explanation of surface characteristics, standards, www.accretech.jp/english/pdf/measuring/sfexplain_e.pdf (assessed 8 July 2012).

[17] B.H. Yan, G.W. Chang, T.J. Cheng, and R.T. Hsu, "Electrolytic magnetic abrasive finishing," Int. J. Mach. Tool. Manuf. vol. 43, pp. 1355-1366, 2003. 